\title{
Effect of Se Isoelectronic Impurity on the Luminescence Features of the $\mathrm{ZnO}$
}

\author{
V. Khomyak ${ }^{a *}$, M. Slyotov ${ }^{a}$, I. Shtepliuk ${ }^{b}$, O. Slyotov $^{a}$ \\ AND V. KOSOLOVSKIY ${ }^{a}$ \\ ${ }^{a}$ Yu. Fedkovich Chernivtsi National University, 58012, Chernivtsi, Ukraine \\ ${ }^{b}$ Frantsevich Institute for Problems of Materials Science, NASU, 03680, Kiev-142, Ukraine
}

\begin{abstract}
Se-doped $\mathrm{ZnO}$ films have been deposited on the sapphire substrates by the radio-frequency magnetron sputtering technique. An influence of the isoelectronic impurity Se on the room-temperature luminescence of the $\mathrm{ZnO}$ films is studied. It is revealed that the Se doping leads to an appearance of the intense near-band edge emission spectrum, which consists of three emission bands. The dominant emission band is related to the recombination of the bound excitons. The radiation caused by the band-to-band transitions of free carriers is observed in the high-energy side of the spectrum $\left(\hbar \omega>E_{\mathrm{g}}\right)$.
\end{abstract}

PACS: 78.55.Et, 78.67.Bf, 81.05.Dz, 81.07.Bc

\section{Introduction}

To realize the high-performance light-emitting devices, an important task is the obtaining the semiconductor materials possessing a high intense of ultraviolet (UV) radiation [1]. Due to its direct and wide bandgap $(3.30 \mathrm{eV})$ and high exciton binding energy $(60 \mathrm{meV})$ at room temperature, $\mathrm{ZnO}$ has attracted considerable attention recently for the fabrication of short wavelength optoelectronic devices [2].

$\mathrm{ZnO}$ thin polycrystalline films are classically deposited by such techniques as magnetron sputtering (DC or RF), chemical vapor deposition, chemical spraying, electron cyclotron resonance plasma sputtering, sol-gel deposition, ion-beam assisted deposition, pulsed laser deposition etc. [3]. However, $\mathrm{ZnO}$ properties, especially luminescence, are strongly affected by deposition method, extrinsic doping and further post-growth treatment. Therefore, the search of the alternative way for the enhancement of the UV emission is vital and crucial.

It is well known that the high-performance near-band edge (NBE) emission may be achieved via doping of isoelectronic impurities $[4,5]$. In this case, the quantum efficiency can be increased up to $15-40 \%$. Recently, we have already investigated the effect of the cadmium isoelectronic impurity on the properties of $\mathrm{ZnO}$ thin films and have revealed that the $\mathrm{Cd}$ content of 0.4 at.\% leads to an increase of the luminescence efficiency [6]. Earlier, it was established that the substitution of a small fraction of the anion atoms with an isoelectronic element of distinctly different electronegativity and/or size leads to

\footnotetext{
* corresponding author; e-mail: V.Khomyak@chnu.edu.ua
}

a dramatic enhancement of the high radiative recombination efficiency $[7,8]$. It is associated with the excitons bound to isoelectronic traps. It is well known that the Auger effect cannot take place for the exciton bound to the isolated isoelectronic traps. So the material with the isoelectronic traps can have the high probability of the radiative optical transitions. Taking into account the above-mentioned, we believe that we can analogously improve the radiative recombination efficiency of $\mathrm{ZnO}$ by replacing a small percentage of oxygen with Se anions.

In this paper we report on the influence of Se isoelectronic impurity on the optical properties of $\mathrm{ZnO}$ thin films obtained by the RF magnetron sputtering.

\section{Experimental details}

Se-doped $\mathrm{ZnO}$ films ( $\mathrm{ZnO}: \mathrm{Se}$ ) have been deposited on the sapphire substrates $\left(c-\mathrm{Al}_{2} \mathrm{O}_{3}\right)$ using the RF magnetron sputtering. High-purity argon was utilized as the working gas. Powder targets (pressured mixture of the zinc and selenium oxides) were used for the sputtering process. The atomic concentration of Se concentration varied from 0.6 to 0.8 at.\% and was determined from the X-ray photoelectron spectroscopy (XPS) peak intensities, similarly to approach, which was presented in [6]. XPS spectra were collected by means of the UHV-Analysis-System SPECS (Germany). A partial pressure of argon in the deposition chamber is maintained at $\approx 0.1 \mathrm{~Pa}$. The target-to-substrate distance was about $40 \mathrm{~mm}$. The film thickness varies in the range of $300-1500 \mathrm{~nm}$ depending on the technological conditions. The crystal structure of the grown films was investigated by the DRON-4 system (operating at $40 \mathrm{kV}$ and $30 \mathrm{~mA}$ ) with the $\mathrm{Cu} K_{\alpha}$ radiation $(\lambda=0.154056 \mathrm{~nm})$ as the source. 
The photoluminescence (PL) measurements were carried out using the conventional installation which operates in two modes: normal and $\lambda$-modulation mode [9]. The main components of this equipment are diffraction monochromator MDR-23 and photomultiplier FEP-79. PL spectra were excited by nitrogen laser operating at the wavelength $\lambda_{\max }$ of $337 \mathrm{~nm}(3.68 \mathrm{eV})$. The resulting signal was registered by the synchronous detecting system and was recorded by the automatic electronic recorder KSP-2. The first-order derivative spectra of the initial spectrum were obtained at the modulation frequency $\Omega$. All studies were performed at the room temperature.

In order to estimate the quantum efficiency $\eta$ the measured intensity of the PL emission of $\mathrm{ZnO}$ :Se films must be compared with the PL intensity of GaAs epitaxial layer. It should be noted that the GaAs layer may be used as a reference sample because its quantum efficiency $\eta$ is $\approx 100 \%$. For such comparisons the spectral sensitivity $S_{\omega}$ of optical system was pre-determined by the classical equation: $S_{\omega}=i_{\omega} / \rho_{\omega}$, where $i_{\omega}$ is the value of the photocurrent of a standard incandescent lamp, $\rho_{\omega}$ is the spectral density of the equilibrium radiation of a standard lamp SI-8-200 (secondary standard), which was calculated by the well-known Planck equation [10]. Photoresponses of the $\mathrm{ZnO}$ :Se and GaAs layers were measured. Using the plotted curve $S_{\omega}$ the values of the PL intensities of $\mathrm{ZnO}$ :Se and $\mathrm{GaAs}$ were defined and their relationship gave us the possibility to estimate of the quantum efficiency $\eta$ of the studied samples.

\section{Results and discussion}

$\mathrm{X}$-ray diffraction (XRD) analysis revealed that all Se-doped $\mathrm{ZnO}$ films crystallize in the hexagonal wurtzitic structure with preferred growth orientation toward the $c$ axis. It should be mentioned that the $\mathrm{ZnO}$ :Se films are characterized by the intense PL band in the wavelength range from $360 \mathrm{~nm}$ to $427 \mathrm{~nm}$ (Fig. 1). The quantum efficiency is about $\approx 15 \%$. When selenium ions are incorporated into $\mathrm{ZnO}$ matrix, the intense NBE emission appears (Fig. 1). Additionally, it was found that the PL intensity for the sample with selenium is 1.5 times higher than the luminescence of undoped $\mathrm{ZnO}$ (Fig. 1, curve 2).

The UV emission band of $\mathrm{ZnO}$ :Se (measured in normal mode) is broadened. Its form and position modify under the changing of the photo-excitation level $L$. It indicates the presence of several components of the luminescence spectra. Figure 2 demonstrates the corresponding differential curve of the emission spectrum (curve 2). Three features are well distinguished and are marked as $A, B$, and $C$ bands (Fig. 2). The origin of these bands may be explained as follows.

The band $A$ is observed in the photon energy range of $\hbar \omega \geq 3.3 \mathrm{eV}$. An analysis of the long-wave absorption edge of obtained $\mathrm{ZnO}$ :Se films gave us the possibility to determine the band gap energy at $300 \mathrm{~K}$ $\left(E_{\mathrm{g}}=3.30 \mathrm{eV}\right)$. This value correlates well with literature

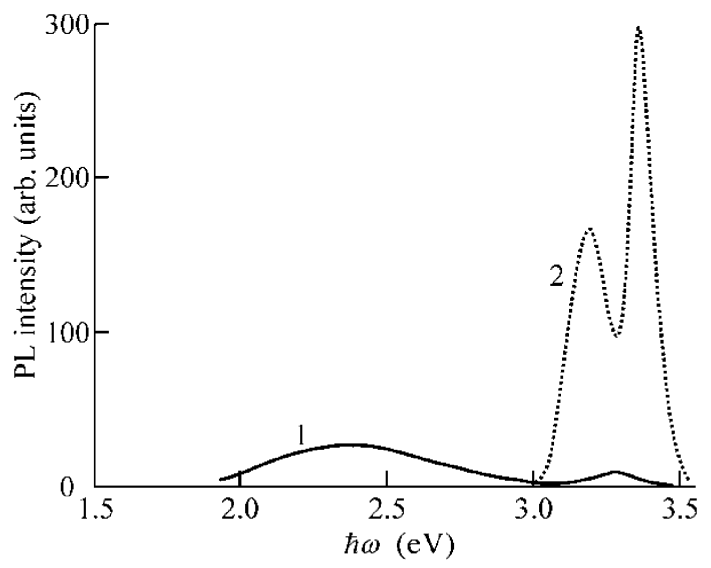

Fig. 1. PL spectra of the undoped (solid curve 1) and Se-doped (dotted curve 2) $\mathrm{ZnO}$ films at the $T=300 \mathrm{~K}$.

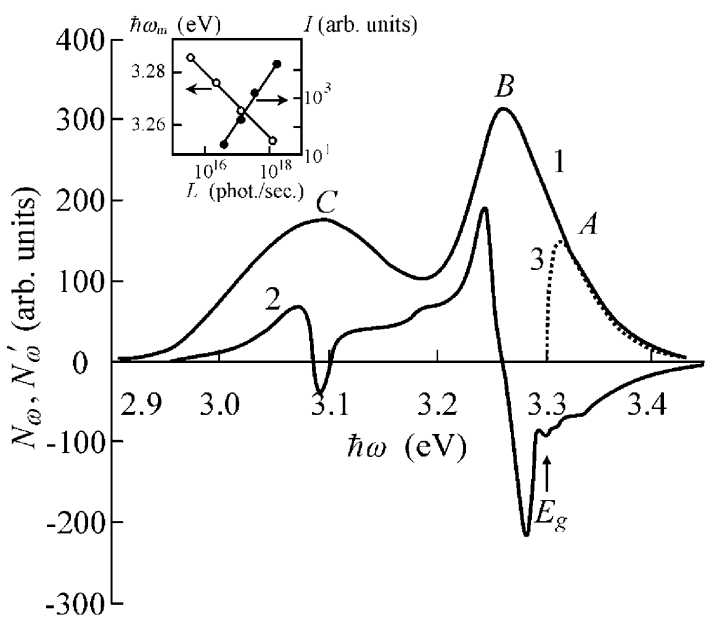

Fig. 2. The normal $N_{\omega}$ (curve 1) and $\lambda$-modulated $N_{\omega}^{\prime}$ (curve 2) PL spectra at the $300 \mathrm{~K}$ as well as a theoretically calculated spectrum of the interband radiation recombination of the free carriers (curve 3) for the $\mathrm{ZnO}: \mathrm{Se}$ thin films. The inset demonstrates the dependences of maximum position $\hbar \omega_{\mathrm{m}}$ and emission intensity $I$ on the level of the photo-excitation $L$.

data [1-3]. Therefore, the emission at the $\hbar \omega \geq E_{\mathrm{g}}$ can be explained by the interband radiative recombination of the free carriers. The corresponding spectral distribution is described by the well-known expression [10]:

$$
N_{\omega} \approx(\hbar \omega)^{2} \sqrt{\hbar \omega-E_{\mathrm{g}}} \exp \left(-\frac{\hbar \omega-E_{\mathrm{g}}}{k T}\right) .
$$

The $N_{\omega}(\hbar \omega)$ curve in the high-energy region was found to be in good agreement with calculations performed by means of the expression (1).

The dominant band is characterized by the following key features:

i) Increasing the excitation level results in a red shift of maximum $\hbar \omega_{\mathrm{m}}$;

ii) The emission intensity varies according to the law of $I \sim L^{1,5}$ (inset in Fig. 2). 
These features are characteristic of the annihilation of excitons upon inelastic scattering at free carriers [11]. Their nature can be explained on the basis of such considerations. The experimental value of binding energy $G_{0}$ can be determined using the magnitude of $E_{\mathrm{g}}-\hbar \omega_{\mathrm{m}}$. The minimum value of the latter is about $\approx 0.049 \mathrm{eV}$. This value is not consistent with the energy of free exciton in $\mathrm{ZnO}(60 \mathrm{meV})[2,3]$. However, using the expression $G_{0}^{\text {theor }}=\left[m_{n}^{*} m_{p}^{*} /\left(m_{n}^{*}+m_{p}^{*}\right)\right] \varepsilon^{-2}$ [12] for calculation of the values of exciton binding energy and effective mass values for electrons $m_{n}^{*}\left(0.28 m_{0}\right)$ and holes $m_{p}^{*}\left(0.78 m_{0}\right)$ as well as dielectric constant $\varepsilon=7.9$ for $\mathrm{ZnO}[1-3]$, we have obtained the value of $G_{0}^{\text {theor }} \approx 44.8 \mathrm{meV}$. The obtained value agrees well with the experimental result obtained in the case of bound exciton.

Let us note that the half-width $\Delta \hbar \omega_{1 / 2}$ of the band $B$ is $\approx 15 \mathrm{meV}$. This value is lower than that of the free exciton $\left(\Delta \hbar \omega_{1 / 2}=k T \approx 26 \mathrm{meV}\right)$. However, the half-width of the band $B$ exceeds the characteristic value for the bound exciton $(0.1 k T)$ [13]. This contradiction can be explained by the presence of the short-range disorder caused by the isoelectronic selenium impurities (ISI), which in turn leads to a local lattice deformation [14]. We believe that, namely, the short-range potential of ISI causes the exciton binding by Se impurity. Additionally, it also promotes the high efficiency excitonic emission and its temperature stability.

The band $C$ with a maximum $\hbar \omega_{\mathrm{m}}$ of $3.1 \mathrm{eV}$ is most probably caused by the recombination via energy level formed by selenium dopant. The depth of the impurity level can be determined on the basis of the experimental results using the energy difference of $E_{\mathrm{g}}-\hbar \omega_{\mathrm{m}} \approx 0.2 \mathrm{eV}$. In general, it is known that the presence of isoelectronic impurities in semiconductors leads to the formation of the donor levels [14]. Using the formula $E_{\mathrm{d}}=G_{0}^{\text {theor }} / 0.2$ [12] we have calculated the binding energy of the exciton bound to donor centers. It was about $\approx 0.22 \mathrm{eV}$. It agrees well with experimental results. An additional confirmation of this result may be obtained by the use of the study of the dependence of the luminescence intensity on the temperature $I(T)$. It is known $[10,13,15]$ that for determining the depth of the respective centers the following expression could be utilized:

$$
I(T)=\frac{I(0)}{1+C_{0} \exp \left(-\frac{E_{\mathrm{T}}}{k T}\right)},
$$

where $I(0)$ is the maximal value of the emission intensity, $C_{0}$ is a constant, $E_{\mathrm{T}}$ is the energy of the thermal activation of the recombination center. In the case of the band $C$, the slope of the straight lines $\ln I(1 / T)$ gave us the possibility to define the $E_{\mathrm{T}}$ value. Our obtained value is about $\approx 0.21 \mathrm{eV}$, which is close to the calculated depth of the donor levels formed by Se impurities.

\section{Conclusions}

In summary, the Se-doped $\mathrm{ZnO}$ films were prepared by means of the RF magnetron sputtering technique. The influence of the isoelectronic selenium impurities on the luminescent properties of $\mathrm{ZnO}$ films was studied and discussed. It was found that the impurity concentration of $0.6-0.8$ at. \% causes the appearance of the intense emission in the spectral range of 350-430 nm with a quantum efficiency of $\approx 15 \%$. Incorporation of Se isoelectronic impurity leads to the formation of donor energy states and localization of excitons by these states. It was determined that the depth of the donor levels formed by Se was about $\approx 0.21 \mathrm{eV}$. The recombination of excitons bound by Se impurities determines the nature of the dominant emission band. These energy states define the recombination processes involving simple centers. Additionally, it was shown that the selenium doping results in the increase of the intensity of the emission band related to the recombination of the free carriers. The effectiveness of this radiation is approximately $\approx 30 \%$ of the total luminescence spectrum.

\section{References}

[1] Y. Chen, D. Bagnall, T. Yao, Mater. Sci. Eng. B 75, 198 (2000).

[2] U. Ozgur, Ja.I. Alilov, C. Lin, A. Teke, M.A. Reshchikov, S. Dogan, V. Avrutin, S.-J. Cho, H. Morkoe, J. Appl. Phys. 98, 041301 (2005).

[3] C. Jagadish, S. Pearton, Zinc Oxide: Bulk, Thin Films and Nanostructures, Elsevier, Oxford 2006.

[4] J.W. Allen, J. Phys. C, Solid State Phys. 4, 1936 (1971).

[5] A. Baldereschi, J. Lumin. 7, 79 (1973).

[6] I. Shtepliuk, G. Lashkarev, O. Khyzhun, B. Kowalski, A. Reszka, V. Khomyak, V. Lazorenko, I. Timofeeva, Acta Phys. Pol. A 120, 500 (2011).

[7] R.A. Faulkner, Phys. Rev. 175, 991 (1968).

[8] A. Baldereschi, J.J. Hopfield, Phys. Rev. Lett. 28, 171 (1972).

[9] V.P. Makhniy, M.M. Slyotov, E.V. Stets, I.V. Tkachenko, V.V. Gorley, P.P. Horley, Thin Solid Films 450, 222 (2004).

[10] V.P. Gribkovskii, The Theory of Light Absorption and Emission in Semiconductors, Nauka i Teknika, Minsk 1975.

[11] E. Koh, D.W. Langer, J. Lumin. 1-2, 514 (1970).

[12] M. Aven, J.S. Prener, Physics and Chemistry of II-VI Compounds, North-Holland, Amsterdam 1967.

[13] P.Y. Yu, M. Cardona, Fundamentals of Semiconductors: Physics and Materials Properties, Springer-Verlag, Berlin 1996.

[14] V.I. Fistul', Atoms in Semiconductors: State and Behaviour, Fizmatlit, Moskva 2004 (in Russian).

[15] J.I. Pankove, Optical Processes in Semiconductors, Prentice-Hall, New Jersey: Inc. Englewood Cliffs 1971. 